\title{
Neutron decay, dark matter and neutron stars
}

\author{
D.H. Beck ${ }^{\mathrm{a}}$ \\ Department of Physics, University of Illinois at Urbana-Champaign, Urbana IL 61801, USA
}

\begin{abstract}
Following up on a suggestion that decay to a dark matter fermion might explain the $4 \sigma$ discrepancy in the neutron lifetime, we consider the implications of such a fermion on neutron star structure. We find that including it reduces the maximum neutron star mass to well below the observed masses. In order to recover stars with the observed masses, the (repulsive) self-interactions of the dark fermion would have to be stronger than those of the nucleon-nucleon interaction.
\end{abstract}

\section{Introduction}

Over the past decade or so, neutron lifetime measurements have settled into two groups whose average values differ by about $4 \sigma$. Lifetime measurements using a beam of neutrons yield an average lifetime of $887.7 \pm 2.2 \mathrm{~s}$, whereas the average result from "bottle" experiments is $878.5 \pm 0.8 \mathrm{~s}$ [1] with two new bottle experiments giving $877.7 \pm 0.7 \pm{ }_{0.2}^{0.4} \mathrm{~s}[1]$ and $881.5 \pm 0.7$ $\pm 0.6 \mathrm{~s}$ [2]. Fornal and Grinstein [3] proposed a possible resolution of the problem: that roughly $1 \%$ of the time neutrons decay to a dark fermion and other standard model or dark sector particles. Dark matter particles, with very weak interactions with standard model particles, would not be among the decay products detected in the beam experiments, whereas they would contribute to the overall decay rate of neutrons as measured in the bottle experiments. A narrow mass range for a dark fermion is, in fact, allowed by the systematics of stable nuclei [3]

$$
\text { 937.9 } \mathrm{MeV}<m_{\chi}<938.7 \mathrm{MeV} \text {; }
$$

in what follows, for simplicity, we will assume $m_{\chi}=m_{n}$ unless explicitly noted.

In addition to studies by two other groups $[4,5]$ that reach similar conclusions, we have considered the impact on the structure of neutron stars [6] of neutron decay to a dark fermion. Neutron stars form, typically, in the aftermath of supernova explosions as the gravitational pressure overcomes the degeneracy pressure of the electrons (just the result of the Pauli exclusion principle) and neutrons (largely) form from the combination of electrons and protons. In neutron stars, the gravitational pressure is balanced both by the neutron degeneracy pressure as well as by the repulsive $n-n$ interaction at high densities. The basic physics scenario we investigate is this: if the neutron were to decay to a dark fermion $1 \%$ of the time, over a time period much shorter than the neutron star lifetime the dark fermions would come to equilibrium and fill their own fermi sea, thereby reducing the number and the Fermi momentum of the neutrons (note that the pressure, $P \propto p_{F}^{5}$ ). Furthermore, if the

a e-mail: dhbeck@illinois .edu dark fermions have weak self-interactions, the substantial additional pressure generated by the repulsive neutronneutron $(n-n)$ interactions would be reduced.

Along with the effect on neutron stars, there has been a study of the impact of a dark decay on the relation between the neutron decay rate and the axial coupling constant $g_{A}$ [7]. Based on the current value of $g_{A}$, this analysis suggests a tension with $1 \%$ of the neutron decay going to a dark channel. There have also been two searches for standard model particles likely to accompany dark fermions in neutron decay. Final states with photons [8] and with $e^{+} e^{-}$[9] have been largely ruled out.

\section{Neutron star physics}

Neutron stars consist of a fluid of interacting neutrons ${ }^{1}$ which we model assuming spherical symmetry, and mechanical and thermal equilibrium at zero temperature. For ordinary stars, mechanical equilibrium is expressed by Newton's second law

$$
\frac{d P}{d r}=-\frac{G}{r^{2}} \rho M(r)
$$

where $P$ is the pressure, $\rho$ is the mass density and $M(r)$ is the mass inside the radius $r$; for neutron stars it is modified to account for the effects of the strong gravitational field. With corrections for general relativity, the Tolman-Oppenheimer-Volkov (TOV) equations obtain $[10,11]$

$$
\frac{d P}{d r}=-\frac{G}{r^{2}} \frac{\epsilon+P}{c^{2}} \frac{m(r)+4 \pi r^{3} P}{c^{2}\left[1-2 G m(r) / r c^{2}\right]},
$$

where $\epsilon(r)$ is the energy density and

$$
m(r)=\int_{0}^{r} d r^{\prime} 4 \pi r^{\prime 2} \epsilon\left(r^{\prime}\right)
$$

is the total energy density contained within the radius $r$.

\footnotetext{
${ }^{1}$ Protons and electrons, in chemical equilibrium with the neutron-typically at a relative concentration of several percent-are included in the equations of state used to model the neutron stars.
} 
The equation of state of neutron matter defines the relation between the pressure and the energy density (for normal stars, e.g., typically the equation of state provides $P(\rho)$ ); we have performed calculations using both a nucleons-only equation of state, AkmalPandharipande-Ravenhall (APR) [12], and an equation of state incorporating deconfined quarks at high densities, a so-called quark-hadron crossover model (QHC18) [13]. Because the densities are high, the Fermi momentum can be large relative the neutron mass, therefore, relativistic kinematics are used. The TOV equations are solved numerically by assuming a central energy density and integrating outward until $P=0$. We are thereby able to calculate the total neutron star mass as a function of central density.

Among the many neutron stars observed, there are two that have particularly well-established masses, PSR J1614-2230 with a mass of $1.928 \pm 0.017$ solar masses $\left(\mathrm{M}_{\odot}\right)[14,15]$ and PSR J0348+0432 with a mass of $2.01 \pm$ $0.04 \mathrm{M}_{\odot}$ [16]. Both are neutron-star-white-dwarf binaries and the masses are determined, in part using the general relativistic time shift of the pulsar signal as it passes the white dwarf (Shapiro delay). It is these observations that set the benchmark of the minimum upper limit for neutron star mass to which we will compare our models. Typically, calculations using nuclear equations of state built from microscopic particle interactions and nuclear properties tend to underpredict the maximum mass; the two equations of state used here are able to generate maximum masses somewhat higher than $2 M_{\odot}$.

For the calculations including dark matter, we assume it is in the form of a non-interacting, relativistic Fermi gas. The energy density in this gas is simply

$$
\epsilon_{\chi}=2 \int_{0}^{k_{F, \chi}} d^{3} k_{\chi} \mu_{\chi}
$$

where $\mu_{\chi}=\sqrt{k_{\chi}^{2}+m_{\chi}^{2}}$ is the dark matter chemical potential, $k_{F, \chi}$ is the dark matter Fermi momentum and the 2 is from the spin degeneracy. Equilibrium in the neutron matter plus dark matter system is determined by the equivalence of their chemical potentials at each radius $\mu_{n}=\mu_{\chi}$, where $\mu_{n}$ is given by the equation of state. This leads to a relation between the Fermi momenta of the neutrons and the dark matter particles, $x=k_{F, \chi} / k_{F, n}$, which is a function of density and hence of the radius within the neutron star. The dark matter energy density and the corresponding pressure, $P_{\chi}=n_{\chi}^{2} \partial\left(\epsilon_{\chi} / n_{\chi}\right) / \partial n_{\chi}$, add to those of the neutrons; for illustration, the standard non-relativistic forms for the total energy and pressure in the system are

$$
\epsilon=\epsilon_{n}\left(\rho_{n}\right)+m_{\chi} \rho_{n} x^{3}+\frac{k_{F, n}^{5}}{10 \pi^{2} m_{\chi}} x^{5},
$$

and

$$
P=P_{n}\left(\rho_{n}\right)+\frac{k_{F, n}^{5}}{15 \pi^{2} m_{\chi}} x^{5} .
$$

The key physics in the problem can be seen in the chemical potential for neutrons as a function of density, Fig. 1. Relative to the energy of a non-interacting fermion, the chemical potential for a neutron is negative for densities near that of nuclear matter, corresponding to

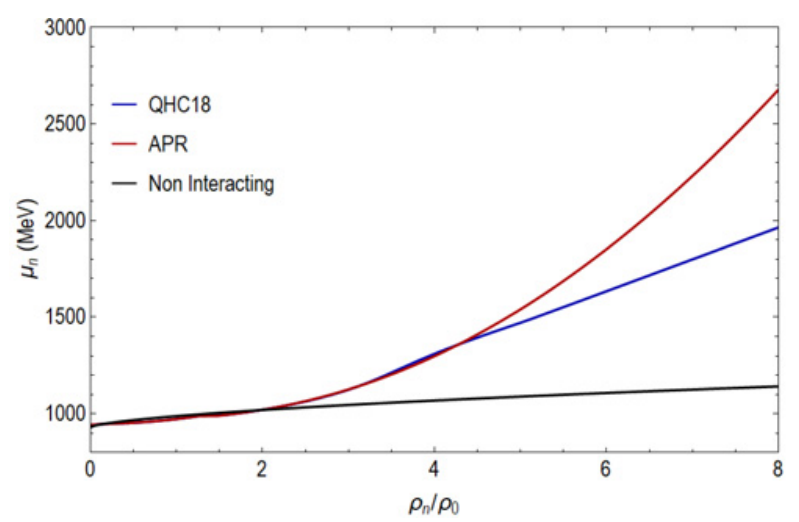

Figure 1. Neutron chemical potential, $\mu_{n}$, (including the rest mass) as a function of the neutron density, $\rho_{n}$, in units of the nuclear saturation density, $\rho_{0}$. As would be expected, the chemical potential for interacting neutrons is lower than that of a non-interacting fermion in the region of nuclear densities. At higher densities, however, because of the hard core of the $n-n$ interaction, the chemical potential is much higher than that of a free fermion gas. In fact the "degeneracy pressure", reflected here by the difference between the non-interacting fermion chemical potential and the rest mass, is relatively small compared to the effect of the hard core for densities $\rho_{n} \gtrsim \rho_{0}$.

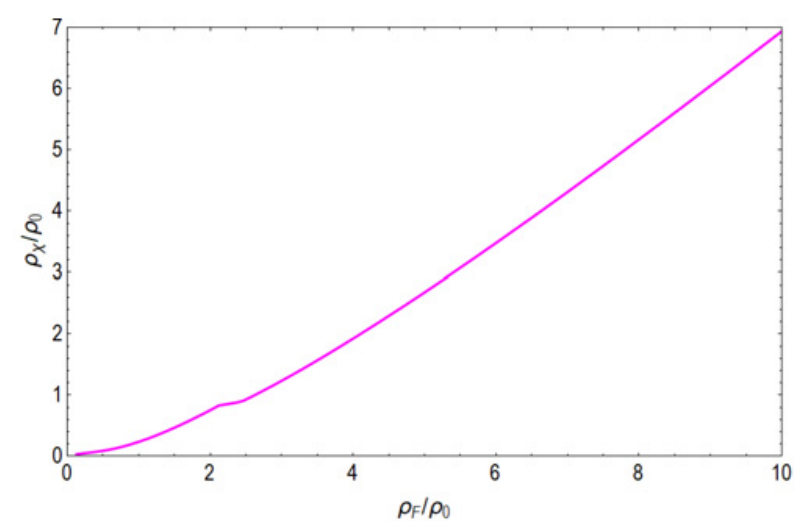

Figure 2. The number of dark matter fermions vs. the total number of fermions (neutrons plus dark fermions) at chemical equilibrium for the QHC18 nuclear equation of state. At twice nuclear matter density, $2 \rho_{0}$, the ratio is about 0.5 and grows to 0.7 at $10 \rho_{0}$. The flat region around $2 \rho_{0}$ corresponds to pion condensation.

the binding of ordinary nuclei. However, as the density increases, the hard core of the $n-n$ interaction dominates and the chemical potential grows to values exceeding twice the neutron mass at the highest densities. Therefore, at these densities, converting neutrons to non-interacting fermions produces a large energy saving.

In more detail, we can ask what the equilibrium ratio of dark matter to neutron matter is as a function of their total density as is shown in Fig. 2. As expected from the discussion above, as the overall density increases, the fraction of dark matter also increases.

\section{Neutron star simulations and implications for neutron decay to dark matter}

In Fig. 3 we show the solutions of the TOV equations in terms of the neutron star mass as a function of central 


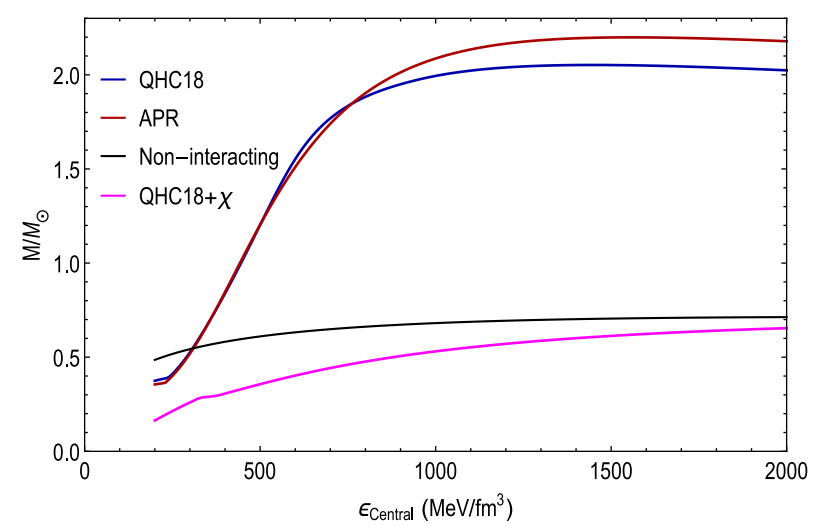

Figure 3. Neutron star masses calculated using the TOV equations for various equations of state: APR [12], QHC18 [13], for non-interacting neutrons and for the QHC18 equation of state with non-interacting dark matter particles in chemical equilibrium. Whereas both the APR and QHC18 equations of state predict maximum neutron star masses consistent with observations, the addition of the non-interacting dark matter degree of freedom reduces the maximum mass to that of the non-interacting bound (at very high central energy densities). Note that the APR $+\chi$ equation of state gives masses only slightly higher than those of $\mathrm{QHC} 18+\chi$. The flat regions at low central density correspond to pion condensation in the underlying nuclear equation of state.

energy density. The nuclear equations of state, APR and QHC18, generate neutron stars with maximum masses greater than $2 M_{\odot}$ as observed. Non-interacting fermions generate a curve with a maximum mass just above $0.7 M_{\odot}$, called the Oppenheimer-Snyder bound [17]. The result for the QHC18 plus dark matter equation of state is easiest to understand at high central densities - as the density increases the matter becomes dominated by its dark component and approaches the non-interacting bound. At low central densities, the physics is dominated by the degeneracy pressure and is reduced by roughly a factor of two as the numbers of neutrons and of dark matter particles are roughly equal. We therefore conclude that neutron decay to dark fermions with no self-interactions is ruled out by the existence of $2 M_{\odot}$ neutron stars.

\section{Further observations}

Whereas this study apparently invalidates the original suggestion of Fornal and Grinstein, it suggests other questions. The possibility of heavier dark fermions produced by a similar mechanism in the environment of a neutron star is investigated in Ref. [6], with the result that, as expected, the only mass that works is $m_{\chi} \gtrsim 2 m_{n}$ such that there are very few dark fermions in the star.
If one solves the problem by invoking interacting dark fermions, the interactions would have to be repulsive and, in fact, stronger those between neutrons in order to overcome in additional the reduction of the degeneracy pressure resulting from the second fermion species. There have been a number of considerations of gravitational capture of dark matter particles by neutron stars [18] and the corresponding limits on the neutron-dark-matter interaction scales-here, the interaction strengths probed by the putative neutron decay, limited only by the neutron star lifetime, are typically much smaller, depending on the particular structure of the dark sector. In the same context, one could also consider the possible coupling of dark matter to strange standard model particles that might exist in the high density regions of neutron starsa coupling that would be very difficult to probe in any other way.

\section{References}

[1] R.W. Patie, et al., Science 360, 627 (2018)

[2] A.P. Serebrov, et al., Phys. Rev. C 97, 055503 (2018)

[3] B. Fornal, B. Grinstein, Phys. Rev. Lett. 120, 191801 (2018)

[4] D. McKeen, A.E. Nelson, S. Reddy, D. Zhou, Phys. Rev. Lett. 121, 061802 (2018)

[5] T.F. Motta, P.A.M. Guichon, A.W. Thomas, J. Phys. G 45, 05LT01 (2018)

[6] G. Baym, D.H. Beck, P. Geltenbort, J. Shelton, Phys. Rev. Lett. 121, 061801 (2018)

[7] A. Czarnecki, W.J. Marciano, A. Sirlin, Phys. Rev. Lett. 120, 202002 (2018)

[8] Z. Tang, et al., (UCNA Collaboration), Phys. Rev. Lett. 121, 022505 (2018)

[9] Z. Sun, et al., (UCNA Collaboration), Phys. Rev. C 97, 052501 (2018)

[10] R.C. Tolman, Phys. Rev. 55, 364 (1939)

[11] J.R. Oppenheimer, G.M. Volkoff, Phys. Rev. 55, 374 (1939)

[12] A. Akmal, V.R. Pandharipande, D.G. Ravenhall, Phys. Rev. C 58, 1804 (1998)

[13] G. Baym, T. Hatsuda, T. Kojo, P.D. Powell, Y. Song, T. Takatsuka, Rep. Progr. Phys. 81, 056902 (2018)

[14] P.B. Demorest, T. Pennucci, S.M. Ransom, M.S.E. Roberts, J.W.T. Hessels, Nature 467, 1081 (2010)

[15] E. Fonseca, et al., ApJ 832, 167 (2016)

[16] J. Antoniadas, et al., Science 340, 1233232 (2013)

[17] J.R. Oppenheimer, H. Snyder, Phys. Rev. 56, 455 (1939)

[18] N. Raj, P. Tanedo, H.B. Yu, Phys. Rev. D 97, 043006 (2018) 